\title{
Tungsten Spectroscopy for Fusion Plasmas
}

\author{
R. Neu ${ }^{1}$, T. Pütterich ${ }^{1}$, R. Dux ${ }^{1}$, A. Pospieszczyk ${ }^{2}$, G. Sergienko ${ }^{2}$, \\ ASDEX Upgrade Team ${ }^{1}$, TEXTOR Team ${ }^{2}$ \\ ${ }^{1}$ MPI für Plasmaphysik, Euratom Association, Boltzmannstr.2, 85748 Garching \\ ${ }^{2}$ Institut für Plasmaphysik, Euratom Association, FZ Jülich GmbH, 52425 Jülich
}

\begin{abstract}
Tungsten is one of very few candidate materials for plasma facing components in future fusion devices. Therefore, investigations have been started at fusion devices and EBITs to provide atomic data for $\mathrm{W}$ in fusion plasmas. Usually the influx of impurities is deduced from the intensity of spectral lines from neutrals or ions in a low ionisation state. For this purpose the appropriate ionisation rates and excitation rates have to be known. At the moment, a WI transition $\left({ }^{7} S-{ }^{7} P\right)$ at $400.9 \mathrm{~nm}$ is used, but an extension of the method to other lines is under investigation. In the core of present day plasmas ionisation states up to $\mathrm{W}^{56+}$ can be reached and in a reactor states up to around $\mathrm{W}^{68+}$ will be present. In order to extract information on the local $\mathrm{W}$ concentrations over the whole plasma radius atomic data (wavelength, excitation, ionisation, recombination) for all the charge states up to the maximum ionisation state are necessary. Similarly, a high sensitivity has to be achieved since the central W concentrations should stay below $10^{-4}$. For an unambiguous identification of the transitions EBIT measurements are of great advantage, but due to the lower electron density compared to fusion plasmas, investigations there are indispensable.
\end{abstract}

PACS: 52.25.Vy, 34.80.Dp, 32.30.Jc, 32.30.Rj, 28.52.-s

Keywords: Tungsten Spectroscopy, High Temperature Plasma, Fusion

\section{Introduction}

The 'International Thermonuclear Experimental Reactor' (ITER), of which the construction will start in 2007, should demonstrate the physical and technological feasibility of fusion for power production [1]. It will employ tungsten at areas with high particle and power load (divertor entrance and baffles) due to its high energy threshold for sputtering and its low sputtering yield compared to the low- $\mathrm{Z}$ materials $\mathrm{C}$ and $\mathrm{Be}$, which will be used in parallel. Although the requirements for the plasma facing components (PFCs) in ITER will already be orders of magnitude larger than in present day devices, the step to a quasi steady state DEMO reactor will still considerably increase the particle fluencies to the PFCs. Therefore it is foreseen that tungsten will be used as armour material for all PFCs [2] in future fusion reactors. 
However, W was already used as plasma facing material (PFM) in early devices, as PLT [3] and ORMAK [4] and it soon became clear that central $\mathrm{W}$ radiation was occasionally as large as the ohmic input power and consequently led to hollow temperature profiles in the plasma discharges [5]. In these cases, the $\mathrm{W}$ concentrations were estimated to be in the range of $10^{-3}[6]$, consistent with estimations using present day atomic data. Such high values would prevent ignition and burning in a fusion reactor as can be deduced form zero dimensional power balance calculations [7]. Unlike these early devices, which used a material limiter to keep the plasma away from the vacuum vessel walls, present day tokamaks use a so called divertor configuration, set up by external magnetic fields allowing to separate the hot bulk plasma from the regions of plasma wall interaction (see for example [8]). By this means the plasma temperature in front of plasma facing components is reduced considerably leading in turn to a strong reduction of the erosion yield for high- $Z$ ions. This opened up again the possibility to use W as a PFM. In order to prepare the plasma physical data base for operation with W PFCs the mid-sized tokamak ASDEX Upgrade [9] has started a dedicated W programme and will be equipped with 100\% W PFCs in the experimental campaign in 2007 [10]. Further investigations are performed in the TEXTOR tokamak [11] and are foreseen in the framework of the ITER-like Wall Project at JET [12], which will use W PFCs in the divertor and Be as PFM in the main chamber. A review on the experiments and results with high-Z plasma facing components can be found in [13].

For these obvious reasons, tungsten is moving back into the focus of spectroscopy for fusion plasmas. The electron temperatures in ITER or a reactor will span from a few eV for the divertor plasma, over $100 \mathrm{eV}$ at the plasma edge up to $25 \mathrm{keV}$ in the core of the plasma. In present day fusion devices, the edge and divertor temperatures are similar, however the central temperatures are usually below $10 \mathrm{keV}$. Therefore $\mathrm{W}$ ionisation states from neutral up to around $\mathrm{W}^{56+}$ exist in present day tokamaks and up to around $\mathrm{W}^{68+}$ will be reached in a reactor.

In most cases spectroscopic investigations in fusion devices are based on measurements along a line of sight (LOS) resulting in integrals of the emissions of ions belonging to many different charge states. This yields complications in linking an experimental spectrum to the presence of a particular ion state in the plasma. Usually, the interpretation can be facilitated using the measured plasma background in connection with transport models. Only recently the process of so called accumulation, a phenomenon, which is common to high- $\mathrm{Z}$ impurities in fusion plasmas with low turbulent transport, was used to restrict the emission to only very few ionisation states [14]. Another experimental technique to produce tungsten ions for spectroscopic investigation is the electron beam ion trap (EBIT, see for example [15]). The EBIT uses a nearly mono-energetic electron beam for the production and excitation of ions. It has the advantage over tokamak-produced plasmas that the charge state distribution of the ions is restricted to only a few states depending on their ionisation potential and can easily be controlled by varying the energy of the ionising electron beam. Similarly to the tokamak the transitions are dominated by electron excitation processes but the electron densities are considerably lower in an EBIT (typically $10^{18} \mathrm{~m}^{-3}$ compared to a fusion plasma with $\approx 10^{20}$ $\mathrm{m}^{-3}$ ) and the electrons are almost mono-energetic. Therefore the transition pattern might 

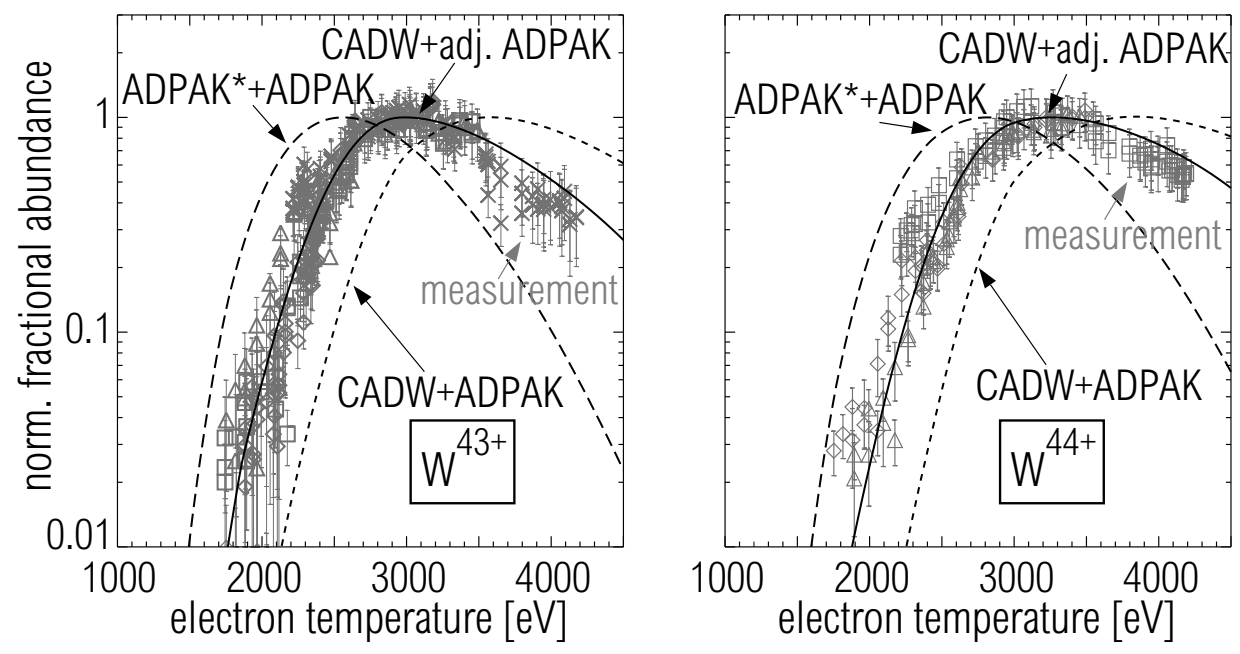

Figure 1: Normalized fractional abundances of $\mathrm{W}^{43+}$ and $\mathrm{W}^{44+}$ deduced from plasma discharges showing a very narrow $\mathrm{W}$ density profile (the symbols represent different discharges). The calculations are given as lines: long dashed (ADPAK $\left.{ }^{*}+\mathrm{ADPAK}\right)$, short dashed (CADW+ADPAK), solid (CADW + adjusted ADPAK). The first term denotes the used ionisation rates, the second one the recombination rates. For more details please see text.

be slightly different. However, an extended operational mode, which simulates the thermal energy distribution by sweeping the energy of the beam was recently successfully employed at the LLNL EBIT [16]. This paper will give an overview on the $\mathrm{W}$ spectroscopy relevant to fusion plasmas. In the next section the methods for the spectroscopic diagnostic of fusion plasmas will shortly reviewed and the third section will deal with the results achieved so far. The final section will assess the available data in view of the future requirements and will emphasize further data needs.

\section{Concepts of Spectroscopic Diagnostic in Fusion Plasmas}

In order to use spectral lines of transitions in atoms or ions in a low charge state as a quantitative measure for the particle influx, the adequate rate coefficients have to be known. Since recombination can be neglected at the edge of low density fusion plasmas, the influx can be determined without detailed knowledge of the local electron density as described by Behringer et al. [17]. Integrating along a line of sight the particle influx $\Gamma_{i n}^{Z}$ from a material surface is given by

$$
\Gamma_{Z}^{i n}=\int_{0}^{R} d l n_{e} S_{(Z, Z+1)} n_{Z}
$$

where $S_{(Z, Z+1)}$ denotes the ionisation rate coefficient for ionisation from charge state $Z$ to $Z+$ 1. In principle, excitations from metastable states within one charge state have to be treated separately, but this is omitted here for simplicity. In the coronal approximation, the collisional electron excitation is balanced by spontaneous emission. Using the usual definition of the 


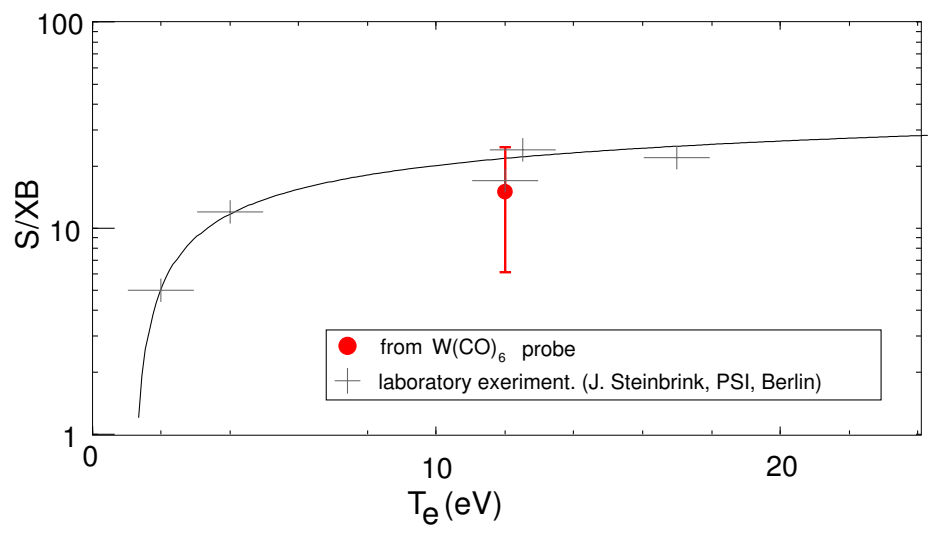

Figure 2: $S / X B$ values of the neutral $\mathrm{W}$ line at $400.9 \mathrm{~nm}$ deduced from measurements in the PSI-1 plasma generator [18] and in ASDEX Upgrade [19].

branching ratio $B$ and integrating over the line of sight on yields the photon flux $\Gamma_{p h, i \rightarrow j}$ for the observed transition:

$$
\Gamma_{p h, i \rightarrow j}=B_{i \rightarrow j} \int_{0}^{R} d l n_{e} X_{i} n_{Z}
$$

with the rate-coefficient $X_{i}$ for the collisional electron excitation. By dividing eq. 1 through eq. 2 one ends up with

$$
\frac{\Gamma_{Z}^{i n}}{\Gamma_{p h, i \rightarrow j}}=\frac{\int_{0}^{R} d \ln _{e} S_{(Z, Z+1)} n_{Z}}{B_{i \rightarrow j} \int_{0}^{R} d l n_{e} X_{i} n_{Z}} .
$$

Since $S_{(Z, Z+1)}$ and $X_{i}$ behave in a very similar way along the line of sight $l$ (similar temperature dependence) and the ion (or atom) with charge $Z$ exists only in a narrow temperature range, one can make the following approximation:

$$
\Gamma_{Z}^{i n}=\frac{S_{(Z, Z+1)}}{B_{i \rightarrow j} X_{i}} \Gamma_{p h, i \rightarrow j}
$$

Therefore, the so called inverse photon efficiency $S / X B$ directly connects the particle flux to the measured photon flux. For transitions with energies close to the ionisation energy, $S / X B$ is only weekly dependent on the electron temperature and under typical conditions (influx of particles against a temperature gradient) $T_{e} \approx 1 / 3 E_{Z}^{i o n}$, with $E_{Z}^{\text {ion }}$ denoting the ionisation potential of the ion with charge $Z$ is a good estimate. In practice, $S_{(Z, Z+1)}$ and $X_{i}$ are often calculated effective rates taking into account also the density dependence. The spectroscopic measurement has to be performed in the direction of the impurity influx and for a laterally non-isotropic particle source the whole emission cloud has to be observed. A recent review on the spectroscopic diagnostic of tokamak edge plasmas is given in [20].

Neglecting plasma transport, the ionisation equilibrium in the core of a fusion plasma is usually well described by the coronal approximation. There, the total ionisation rate is dominated by direct electron ionisation and contributions from auto-ionisation of inner shell excited states. The total recombination rate is mainly given by radiative recombination which is most important for highly charged ions with only few electrons remaining. In the process of 
dielectronic recombination, the energy of the electron is transferred to the recombining ion leading to double excitation, which is stabilised by a radiative transition. Usually, the stabilisation process is more likely than the auto-ionisation process for highly charged ions, since the relaxation rates increase with $Z^{4}$. Due to the high actual importance of $\mathrm{W}$ in fusion plasmas all the necessary rates are presently revisited and recalculated [21] within the framework of the ADAS database [22]. Figure 1 shows the measured fractional abundance of $\mathrm{W}^{43+}$ and $\mathrm{W}^{44+}$ together with calculations using different rates for ionisation and recombination [23] as will be explained in the next section.

Summarising, the continuity equations in coronal equilibrium can be written as a set of $Z$ equations of the form:

$$
\frac{\partial}{\partial t} n_{Z}+\nabla \vec{\Gamma}_{Z}=n_{e}\left(n_{Z-1} S_{Z-1}+n_{Z+1} \alpha_{Z+1}-n_{Z} S_{Z}-n_{Z} \alpha_{Z}\right)
$$

with the particle flux $\Gamma_{Z}$ to take into account transport effects. Usually it is described by using a diffusive and convective term

$$
\vec{\Gamma}_{Z}=D_{Z} \nabla n_{Z}+v_{Z} n_{Z}
$$

For charge state resolved transport calculation as they are necessary for the interpretation of spectroscopic measurements, $Z$ coupled equations of the form of eq. 5 have to be solved taking. This is done numerically using a transport code as for example STRAHL [24, 25] or MIST [26]. The particle transport in fusion plasma is still a matter of current research. It depends strongly on the background plasma parameters and is usually divided in terms caused by particle collisions (neoclassical transport) and fluctuations (anomalous transport). The neoclassical transport parameters $\left(D_{Z}^{n e o}, v_{Z}^{n e o}\right)$ show a pronounced $Z$ dependence and therefore are of special interest for the description of the impurity transport. A recent comprehensive overview of experimental results for impurity transport is given in [27]. For the investigations presented here, it is important to note that the impurity transport in the centre of fusion plasma usually is very small. Therefore the influence on the deduced quantities, as for example the ionisation equilibrium, is quite low. However, even small drifts $\left(v_{Z}\right)$ can change the total impurity density profile, which has to be taken into account when interpreting spectroscopic signals.

As in the case of influx measurements, also the central measurements are usually LOS based, similar as described in eq.2. Generally, fusion plasmas show a monotonically increasing electron temperature from the plasma edge towards its centre, leading to a shell like structure of the ionisation states with an increasing charge number from the edge to the centre. In principle, this can be reconstructed from independent measurements of the background plasmas, when the adequate atomic physics data as well as the transport parameters are at hand. 


\begin{tabular}{rrrcccccr}
\hline$\lambda(\mathrm{nm})$ & \multicolumn{2}{c}{$E\left(\mathrm{~cm}^{-1}\right)$} & \multicolumn{2}{c}{$g_{L}$} & \multicolumn{2}{c}{$\operatorname{transition}$} & $A\left(\mathrm{~s}^{-1}\right)$ & br $(\%)$ \\
& low & up & low & up & low & up & & \\
\hline 255.135 & 0.00 & 39183.20 & 0.00 & 1.00 & $\mathrm{a}_{-}{ }^{5} D_{0}$ & $\mathrm{x} J=1$ & $1.8 e+8$ & 86 \\
2681.42 & 2951.29 & 40233.97 & 1.98 & 1.50 & $\mathrm{~b}_{-}{ }^{7} S_{3}$ & $\mathrm{x} J=4$ & $7.4 e+7$ & 86 \\
400.875 & 2951.29 & 27889.68 & 1.98 & 1.70 & $\mathrm{~b}_{-}{ }^{7} S_{3}$ & $\mathrm{~d}_{-}{ }^{7} P_{4}$ & $1.6 e+7$ & 99 \\
429.461 & 2951.29 & 26229.77 & 1.98 & 1.84 & $\mathrm{~b}_{-}{ }^{7} S_{3}$ & $\mathrm{~d}_{-}{ }^{7} P_{2}$ & $1.2 e+7$ & 94 \\
488.690 & 6219.33 & 26676.48 & 1.50 & 1.46 & $\mathrm{a}_{-}^{5} D_{4}$ & $\mathrm{c}_{-}{ }^{7} F_{5}$ & $8.1 e+5$ & 100 \\
498.259 & 0.00 & 20064.30 & 0.00 & 1.54 & $\mathrm{a}_{-}^{5} D_{0}$ & $\mathrm{c}_{-}{ }^{7} F_{1}$ & $4.2 e+5$ & 79 \\
505.328 & 1670.29 & 21453.90 & 1.51 & 2.51 & $\mathrm{a}_{-}{ }^{7} D_{1}$ & $\mathrm{c}_{-}^{7} D_{1}$ & $1.9 e+6$ & 52 \\
\hline
\end{tabular}

Table 1: Selected lines of neutral $\mathrm{W}$ for influx measurements in fusion devices from [28]. Abbreviations: $\mathrm{a}=5 d 4\left({ }^{5} D\right) 6 s 2, \mathrm{~b}=5 d 5\left({ }^{6} S\right) 6 s, \mathrm{c}=5 d 4\left({ }^{5} D\right) 6 s 6 p, \mathrm{~d}=5 d 5\left({ }^{6} S\right) 6 p$, x means unidentified.

\section{W spectroscopy in fusion plasmas}

\subsection{W Influx Measurements}

A thorough diagnostic of the $\mathrm{W}$ influx into the plasma is of outmost importance in order to interpret the $\mathrm{W}$ behaviour in the plasma. However, theoretical data for the inverse photon efficiency $S / X B$ are scarce or even missing in the case of tungsten. There exists only an experimental dataset from the former PSI-1 plasma generator device (Berlin) for a WI transition $\left(5 d^{5} 6 s 7 S_{3}-5 d^{5} 6 p^{7} P_{4}\right)$ at $400.875 \mathrm{~nm}$ [18] which was verified at ASDEX Upgrade under fusion plasma conditions [19] (see Fig.2). Recently, a comprehensive effort has been started at the tokamak TEXTOR [28] to compare experimental spectroscopic results with those calculated from the ATOM code [29], in order to provide reliable data for the determination of tungsten fluxes and to extent the $S / X B$ method to other transitions. This is especially important because the wavelength of the above mentioned line does not allow long transfer line via fibres. Table 1 presents the lines taken from the latest NIST table [30] which are identified to be best suited for influx measurements.

\subsection{W Density Measurements}

Spectral lines from highly charged tungsten ions have been found in the VUV at least since the use of a W limiter in PLT and ORMAK [5, 6, 31]. However, concentrations of W in fusion plasmas were mostly calculated from the radiation loss parameter from an average ion model $[32,33]$. Therefore quantitative spectroscopy has to be developed to extract the $\mathrm{W}$ densities from spectroscopic signatures. Ideally, lines from all existent ionisation states should be evaluated in order to compensate for the missing radial resolution of line of sight measurements as they are usually performed in spectroscopy. According to the temperature, which could be achieved in the tokamaks of the 70ties, the first spectral features identified in fusion plasmas originated from charge states from Ag-like $\mathrm{W}^{27+}$ to $\mathrm{Y}$-like $\mathrm{W}^{35+}$ and are known as the $\mathrm{W}$ quasicontinuum $[6,31])$. A more recent study of the tungsten impurity spectrum at the ASDEX 


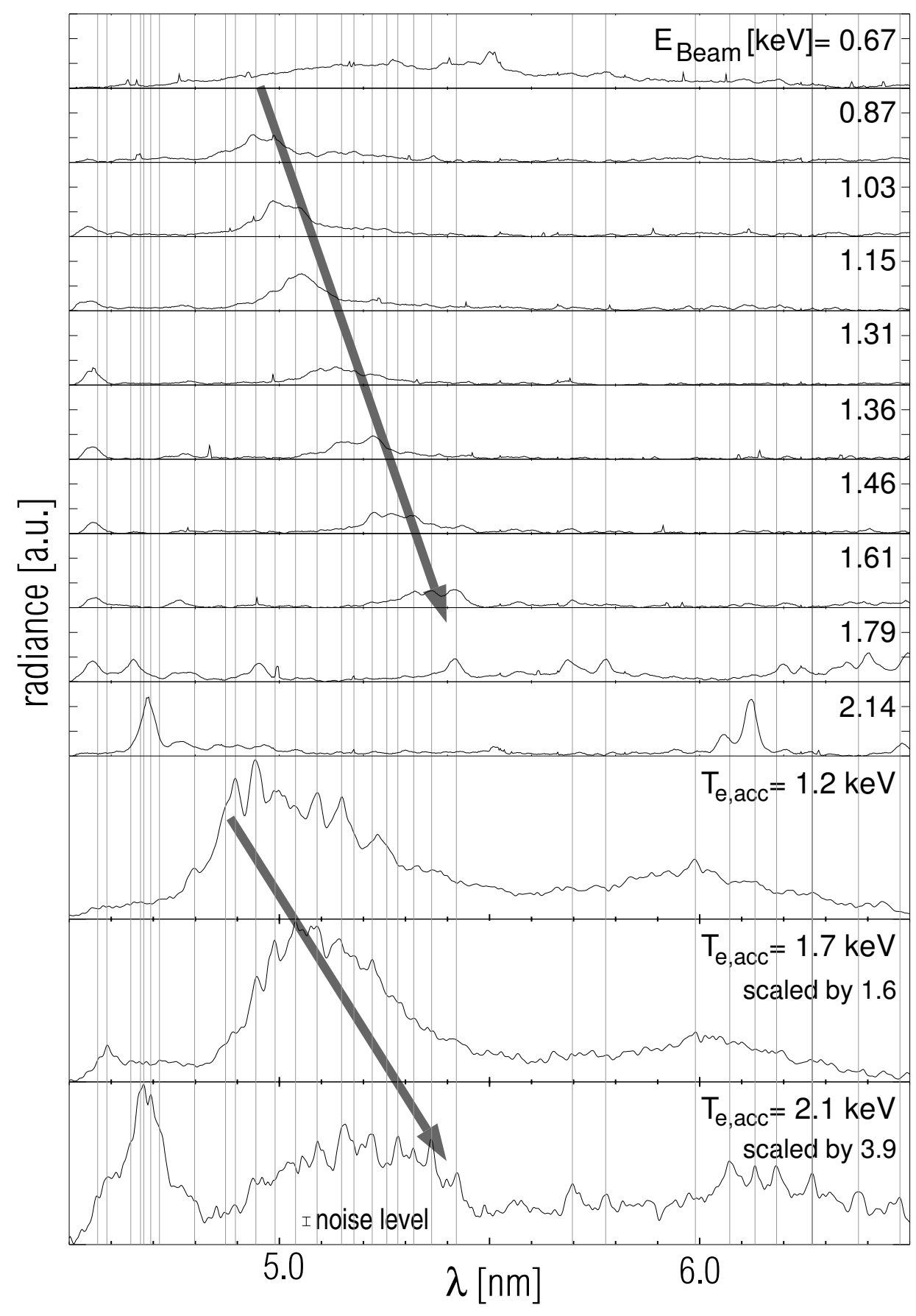

Figure 3: Comparison of EBIT spectra for several electron beam energies with tokamak spectra from an ASDEX Upgrade discharge with impurity accumulation for different central electron temperatures $T_{e, a c c}$ [14]. Vertical lines are drawn to guide the eye. The tokamak spectra are scaled relatively to the spectrum taken at $T_{e, a c c}=1.2 \mathrm{keV}$. 
Upgrade tokamak [34] reported that for electron temperatures above $2 \mathrm{keV}$, many isolated spectral lines appear in the spectrum which superimpose the quasicontinuum emission band. These lines represent transitions in the higher charged tungsten ions (up to $\mathrm{Cu}$-like $\mathrm{W}^{45+}$ ). Motivated by the needs of the fusion community measurements of the VUV and SXR (see below) radiation from tungsten ions have been performed at the Berlin [35] and the Lawrence Livermore National Laboratory [36] EBITs. In [35] spectra were recorded for more than 20 different ions ranging from I-like $\mathrm{W}^{21+}$ to $\mathrm{Cu}$-like $\mathrm{W}^{45+}$, while in [36] the narrower range from $\mathrm{Rb}$-like $\mathrm{W}^{37+}$ to $\mathrm{Cu}$-like $\mathrm{W}^{45+}$ was covered. As already mentioned, these measurements allow restricting the simultaneously observed ion states to a rather low number, which facilitates the identification of transition using atomic physics codes as HULLAC [37, 38] or the Cowan code [39] considerably. The upper part of Fig. 3 presents spectra recorded at different energies of the electron beam in the Berlin EBIT [35]. At the lowest energy ionisation states up to $\mathrm{W}^{25+}$ can be reached whereas at $E_{\text {Beam }}=2.1 \mathrm{keV}$ ions with charges states around $\mathrm{W}^{42+}$ are present. The arrows mark the distinct shift of the centre of the quasi-continuous structure at 5.0-5.3 $\mathrm{nm}$ towards longer wavelengths when increasing the beam energy / $\mathrm{W}$ charge state. One should note that although only a few charge states $(\approx 2-4)$ are mainly contributing to each spectrum, no strong single lines can be identified within the resolution of the spectrometer. Only at the highest beam energies single lines appear which originate from $\mathrm{W}^{39+}-\mathrm{W}^{45+}$ [35]. Using discharges in tokamak ASDEX Upgrade, which exhibit impurity accumulation a reduction of contributing charge states could also be achieved recently [14]. In the well-characterized phenomenon of impurity accumulation [40], the tungsten impurity density in the centre of the plasma (i.e. less than $20 \%$ of plasma radius) peaks by a factor as large as 20, due to neoclassical transport effects. As a result the measured spectra are dominated by the emissions in this narrow region with small radial variations in electron temperatures $\left(T_{e}= \pm 200 \mathrm{eV}\right)$. Additionally, temporal variations of the central temperature allow to scan different ionisation states. This is shown in the lower part of Fig. 3, where three spectra at different central temperatures $\left(T_{e, a c c}\right)$, where accumulation appeared, are presented. Similar as in the EBIT spectra the distinct shift of the centre of gravity as well as the appearance of single lines can be observed. However, the broad structure appearing at $6 \mathrm{~nm}$, which is typical for the $\mathrm{W}$ quasicontinuum measured in tokamak plasmas, does not show up in the EBIT spectra. These obvious difference is possibly related to the higher electron densities in the tokamak compared to the EBIT, which could enhance many weak spectral lines as it is indicated in the calculations presented in [35]. For more details the reader should refer to [14].

Accumulation discharges were also used to deduce the temperature dependence of the fractional abundances of $\mathrm{W}^{27+}-\mathrm{W}^{46+}\left(\mathrm{W}^{43+}\right.$ and $\mathrm{W}^{44+}$ are presented as an example in Fig. 1). Here one uses additionally the fact that the emissivity of a VUV spectral line only weekly depends on the plasma temperature, but is strongly related to the abundance of the related ion state, because the excitation rate is almost constant over the temperature range where the ion exists $[14,23]$. By variing $T_{e, a c c}$ in the accumulation zone, the temperature dependence of the fractional abundance can be deduced after corrections for changes in the total $\mathrm{W}$ density. When comparing the results of the measurements to calculations using ionisa- 
tion/recombination rates from ADPAK [32], where the ionisation rates were corrected for excitation auto-ionisation [34], a strong temperature shift in the equilibrium is found. Using the latest ionisation rates from configuration average distorted wave (CADW) calculations [41] shifts the calculated fractional abundances to too high temperatures. Finally the solid lines represent calculations where the recombination rates where adjusted (adjusted ADPAK) to reproduce the experimental findings [23].

Similarly to the advances in the VUV spectral range, a lot of new data have been produced in the SXR spectral range. Here, usually transitions with $\Delta n \geq 1$ are observed, making the atomic physics calculations much more reliable. A comprehensive compilation of transitions within $\mathrm{W}^{27+}-\mathrm{W}^{46+}$ from ab initio calculations with the HULLAC code is found in [42]. Generally, the wavelength as well as the strength of the single spectral lines compare well to the measurements from the ASDEX Upgrade tokamak [43, 44, 45, 21, 23], where SXR spectral lines originating from $\mathrm{W}^{39+}-\mathrm{W}^{49+}$ have been identified in the spectral range from 0.4 $2.0 \mathrm{~nm}$. The assignement of the transitions is supported by EBIT experiments which were performed at the LLNL, with electron beam energies up to $4.6 \mathrm{keV}$ recording spectra in the 0.5-0.6 nm range [46].

The measurements both from the VUV and the SXR spectral regions are routinely used to derive $\mathrm{W}$ density profiles in ASDEX Upgrade discharges (see for example [10]). This can be achieved by comparing the emissions from different charges states as indicated in the second section.

\section{Conclusions and Outlook}

Large progress on the $\mathrm{W}$ spectroscopy in fusion devices has been made since the early investigations in tokamaks. This encourages the use of the developed tools to extrapolate from the current experimental findings to spectra as they might be observed in future experiments. Fig. 4 shows synthetic spectra resulting from STRAHL calculations using atomic data from the Cowan code and the adjusted rates for ionisation/recombination as described in the previous section. The given plasma parameters $\left(T_{e}, n_{e}\right.$ and the length of the LOS $)$ are typical values for the existing devices ASDEX Upgrade and JET as well as the foreseen values for ITER. The calculations are performed assuming a radially uniform $\mathrm{W}$ concentration. They reveal that the spectra at JET are expected to be rather similar to ones observed at ASDEX Upgrade. Together with the predicted spectral radiance these calculations can largely facilitate the design of a $\mathrm{W}$ diagnostic for the ITER-like Wall project at JET. For the experimental conditions expected in ITER, additional lines will evolve at about $0.15 \mathrm{~nm}$ originating from $\mathrm{W}^{57+}-\mathrm{W}^{67+}$, which should be used as a diagnostic for the central $\mathrm{W}$ content.

However the extrapolation to ITER is quite far and no fusion device is at hand to bridge this gap. Therefore the EBITs offer a unique tool to explore the spectroscopic signatures of $\mathrm{W}$ even for highest ionisation states reachable in future fusion reactors. Equally important is the production and benchmarking of atomic data for the interpretation of the measured spectra. Although high accuracy of the wavelengths is always preferable for their unambiguous identification, the situation is partly alleviated by the fact that the variation of the experimental 


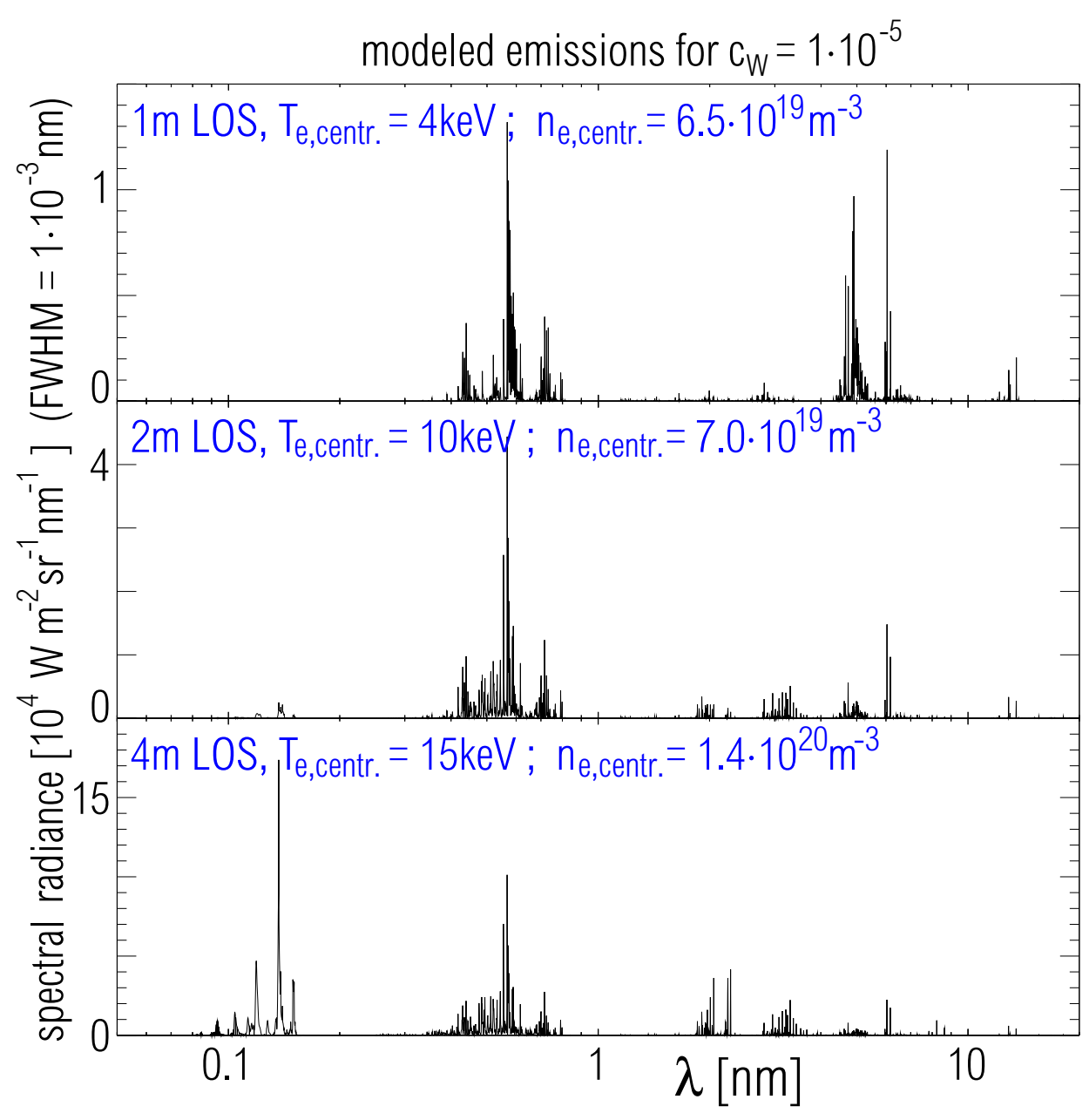

Figure 4: Predicted spectra in the VUV and the SXR spectral region for typical conditions in the tokamaks ASDEX Upgrade, JET and ITER (top to bottom). Note that the overall structures and the absolute intensities agree very well with measurements in the case of ASDEX Upgrade. 
parameters can supply further boundary conditions for the identifications of spectral lines. Under the premises of using spectroscopy as quantitative tool, the excitation rates and in the case of non-resonant transitions, even the setup of a collisional radiative model is necessary. However, not only the rates within one ionisation state need a careful revisiting but also the ionisation and recombination rates as could be extracted from tokamak measurements and in EBIT investigations on the ionisation equilibrium. Besides the highest charge states of $\mathrm{W}$ also the ionisation states with low charge numbers should be adressed. At the moment there is a huge gap in the observed ionisation states between the neutral or singly ionized tungsten at the very edged and the ions which emit the quasicontinuum. The measurement of the charge states in-between would allow to investigate the behaviour of the $\mathrm{W}$ influx in more detail. The final goal of the investigations will be the incorporation of all available data in a data structure like ADAS to make them most commonly available in the fusion community.

\section{References}

[1] R. Aymar, P. Barabaschi, and Y. Shimomura, Plasma Phys. Control. Fusion 44 (2002) 519.

[2] H. Bolt, V. Barabash, W. Krauss, J. Linke, R. Neu, et al., J. Nucl. Mater. 329-333 (2004) 66.

[3] J. Hosea, R. Goldston, and P. Colestock, Nucl. Fusion 25 (1985) 1155.

[4] ORMAK-ISX Group, Nucl. Fusion 25 (1985) 1137.

[5] V. Arunasalam, C. Barnes, K. Bol, K. Brau, N. Bretz, et al., in Proc. 8th Conf. EPS, Prague 1977, volume 2, pages 17-28, Geneva, 1978, EPS.

[6] E. Hinnov, K. Bol, D. Dimock, R. Hawryluk, D. Johnson, et al., Nucl. Fusion 18 (1978) 1305 .

[7] R. Neu, R. Dux, A. Geier, O. Gruber, A. Kallenbach, et al., Fus. Eng. Design 65 (2003) 367.

[8] J. Wesson, Tokamaks, Clarendon Press, Oxford, 3 edition, 2003.

[9] A. Herrmann and O. Gruber, Fusion Science and Technology 44 (2003) 569.

[10] R. Neu, R. Dux, A. Kallenbach, T. Pütterich, M. Balden, et al., Nucl. Fusion 45 (2005) 209.

[11] U. Samm, Fus. Science Technol. 47 (2005) 73.

[12] J. Pamela, G. Matthews, V. Philipps, and R. Kamendje, J. Nucl. Mater. 363-365 (2007) 1 . 
[13] R. Neu, Physica Scripta T123 (2006) 33.

[14] T. Pütterich, R. Neu, C. Biedermann, R. Radtke, and ASDEX Upgrade Team, J. Phys. B: At. Mol. Opt. Phys. 38 (2005) 3071.

[15] J. Gillaspy, Trapping of highly Charged Ions: Foundamentals and Applications, NOVA Science Publishers Inc., ISBN 1-56072-725-X, 2001.

[16] K. Wong, M. May, P. Beiersdorfer, K. Fournier, B. Wilson, et al., Phys. Rev. Let. 90 (2003) 235001.

[17] K. Behringer, H. Summers, B. Denne, M. Forrest, and M. Stamp, Plasma Phys. Control. Fusion 31 (1989) 2059 .

[18] A. Thoma, K. Asmussen, R. Dux, K. Krieger, A. Herrmann, et al., Plasma Phys. Control. Fusion 39 (1997) 1487.

[19] A. Geier, H. Maier, R. Neu, K. Krieger, and ASDEX Upgrade Team, Plasma Phys. Control. Fusion 44 (2002) 2091.

[20] A. Pospieszczyk, Physica Scripta T119 (2005) 71 .

[21] T. Pütterich, Technical Report 10/29, IPP, Garching, Germany, 2006.

[22] H. P. Summers, Atomic data and analysis structure users manual, JET-IR 06 (Abingdon: JET Joint Undertaking) (1994).

[23] T. Pütterich, R. Neu, R. Dux, A. Kallenbach, C. Fuchs, et al., in submitted to Plasma Phys. Control. Fusion, 2006.

[24] K. Behringer, Technical Report JET-R(87)08, JET Joint Undertaking, Abingdon, United Kingdom, 1987.

[25] R. Dux, Rep. IPP 10/30, Max-Planck-Institut für Plasmaphysik, Garching, 2006.

[26] R. Hulse, Nucl. Technol. Fusion 3 (1983) 259.

[27] R. Dux, Rep. IPP 10/27, Max-Planck-Institut für Plasmaphysik, Garching, 2004.

[28] I. Beigmann, G. Sergienko, L. Vainshtein, A. Pospieszczyk, et al., in to be submitted to Plasma Phys. Control. Fusion, 2006.

[29] W. Wiese, M. Smith, and B. Glennon, Technical Report NSRDS-N.B.S. 4, Vol.1, 1966.

[30] Y. Ralchenko, J. Fuhr, F.-C. Jou, et al., in Atomic and Molecular Data and Their Applications, AIP Conference Proc., edited by E. Kato, H. Funaba, and D. Kato, volume 771, pages 276-285, Melville, N.Y., 2005, AIP Press.

[31] R. Isler, R. Neidigh, and R. Cowan, Phys. Lett. A63 (1977) 295. 
[32] D. Post, R. Jensen, C. Tarter, W. Grasberger, and W. Lokke, At. Data Nucl. Data Tables 20 (1977) 397.

[33] D. Post, J. Abdallah, R. Clark, and N. Putvinskaya, Phys. Plasmas 2 (1995) 2328 .

[34] K. Asmussen, K. B. Fournier, J. M. Laming, R. Neu, J. F. Seely, et al., Nucl. Fusion 38 (1998) 967.

[35] R. Radtke, C. Biedermann, J.-L. Schwob, P. Mandelbaum, and R. Doron, Phys. Rev. A 64 (2001) 012720.

[36] S. B. Utter, P. Beiersdorfer, and E. Träbert, Can. J. Phys. 80 (2002) 1503.

[37] M. Klapisch, Comp. Phys. Commun. 2 (1971) 239.

[38] A. Bar-Shalom and M. Klapisch, Comp. Phys. Commun. 50 (1988) 375.

[39] R. Cowan, in Univ. of Calif. Press, 1981.

[40] R. Dux, A. G. Peeters, A. Gude, A. Kallenbach, R. Neu, et al., Nucl. Fusion 39 (1999) 1509.

[41] S. D. Loch, J. A. Ludlow, M. S. Pindzola, A. D. Whiteford, and D. C. Griffin, Physical Review A (Atomic, Molecular, and Optical Physics) 72 (2005) 052716.

[42] K. Fournier, At. Data Nucl. Data Tables 68 (1998) 1 .

[43] R. Neu, K. B. Fournier, D. Schlögl, and J. Rice, J. Phys. B: At. Mol. Opt. Phys. 30 (1997) 5057, preprint in IPP 10/7, June 1997.

[44] R. Neu, K. B. Fournier, D. Bolshukhin, and R. Dux, Physica Scripta T92 (2001) 307.

[45] R. Neu, Technical Report 10/25, IPP, Garching, Germany, 2003.

[46] P. Neill, C. Harris, A. Safronova, S. Hamasha, S. Hansen, et al., Can. J. Phys. 82 (2004) 931. 